\title{
Study Site Number
}

National Cancer Institute

\section{Source}

National Cancer Institute. Study Site Number. NCI Thesaurus. Code C83100.

The numerical characters used to identify, name, or characterize the study site. 